\title{
A new look at the Jordan-Hölder theorem for semimodular lattices
}

\author{
G. GrätZer And J.B. NATION
}

\begin{abstract}
We show that in a semimodular lattice $L$ of finite length, from any prime interval we can reach any maximal chain $C$ by an up- and a down-perspectivity. Therefore, $C$ is a congruence-determining sublattice of $L$.
\end{abstract}

A classical theorem of R. Dedekind [2] (see also R. Dedekind [3]) states that the factors of any chief series (maximal chain of normal subgroups) of a finite group are invariant. C. Jordan, O. Hölder, and H. Wielandt generalized this result to the factors of any composition series (maximal chain of subnormal subgroups).

Formulations of this result for modular lattices are well-known; they are called the Jordan-Hölder Theorem. For semimodular lattices of finite length, most books (see, for instance [1, page 40], [4, page 226]) contain only a much weaker result: any two maximal chains in a semimodular lattice have the same length.

There is a stronger version, however, for semimodular lattices that requires only a slight extension of Dedekind's original argument. This can be found in $[6$, page 444] or [7, Chapter 9]. (Recall that the intervals $[a, b]$ and $[c, d]$ are perspective if $b \vee c=d$ and $b \wedge c=a$ or vice versa. Projectivity is the transitive closure of perspectivity.)

Theorem 1. In a semimodular lattice, let

$$
\begin{aligned}
& C: 0=c_{0} \prec c_{1} \prec \cdots \prec c_{n}=1, \\
& D: 0=d_{0} \prec d_{1} \prec \cdots \prec d_{n}=1 .
\end{aligned}
$$

Then there is a permutation $\pi$ of the set $\{1, \ldots, n\}$ such that $\left[c_{i-1}, c_{i}\right]$ is projective to $\left[d_{\pi(i)-1}, d_{\pi(i)}\right]$, for all $i$.

In this note, we improve on Theorem 1 by replacing projectivity with two perspectivities.

Theorem 2. In a semimodular lattice, let

$$
\begin{aligned}
& C: 0=c_{0} \prec c_{1} \prec \cdots \prec c_{n}=1 \\
& D: 0=d_{0} \prec d_{1} \prec \cdots \prec d_{n}=1 .
\end{aligned}
$$

Date: June 7, 2009.

2000 Mathematics Subject Classification: Primary: 06C10; Secondary: 06B05.

Key words and phrases: Semimodular lattice, maximal chain, finite length, congruencedetermining sublattice. 
Then there is a permutation $\pi$ of the set $\{1, \ldots, n\}$ with the following property: there exists a (prime) interval $\mathfrak{p}_{i}$ such that $\left[c_{i-1}, c_{i}\right]$ is up-perspective to $\mathfrak{p}_{i}$ and $\mathfrak{p}_{i}$ is down-perspective to $\left[d_{\pi(i)-1}, d_{\pi(i)}\right]$, for all $i$.

Remark. Recall that the lattice of subnormal subgroups of a finite group is lower semimodular, so the dual of this theorem applies to yield the full Jordan-Hölder theorem.

Proof. By induction on $\operatorname{len}(L)$. The statement is obvious for $\operatorname{len}(L) \leq 2$, so let $\operatorname{len}(L)>2$.

Let $k$ be the largest integer with $c_{1} \not \leq d_{k}$; note that $k<n$. If $k=0$, then $c_{1}=d_{1}$ and the statement follows by the induction hypothesis. So we can assume that $k>0$.

For $0 \leq j \leq n$, let $e_{j}=c_{1} \vee d_{j}$. Note that $e_{0}=c_{1}$ and $e_{k}=e_{k+1}=d_{k+1}$, and indeed $e_{j}=d_{j}$ for $j \geq k+1$. Now

$$
c_{1}=e_{0} \prec e_{1} \prec \cdots \prec e_{k}=e_{k+1} \prec e_{k+2} \prec \cdots \prec e_{n}=1
$$

is a maximal chain in the interval $\left[c_{1}, 1\right]$. By induction, there is an bijective map $\sigma:\{2, \ldots, n\} \rightarrow\{1, \ldots, k, k+2, \ldots, n\}$ such that, for $i>1$, each interval $\left[c_{i-1}, c_{i}\right]$ is up-perspective to some prime interval $\mathfrak{p}_{i}$ in $L$, which in turn is down-perspective to $\left[e_{\sigma(i)-1}, e_{\sigma(i)}\right]$. For $j \leq k,\left[e_{j-1}, e_{j}\right]$ is down-perspective to $\left[d_{j-1}, d_{j}\right]$, while for $j>k+1$ we have $\left[e_{j-1}, e_{j}\right]=\left[d_{j-1}, d_{j}\right]$. Meanwhile, $\left[0, c_{1}\right]$ is is up-perspective to $\left[d_{k}, d_{k+1}\right]$. So we may take $\pi$ to be the permutation with $\pi(i)=\sigma(i)$, for $i \neq 1$, and $\pi(1)=k+1$.

Given a sublattice $S$ of a lattice $L$, we say that $S$ is a congruence-determining sublattice of $L$ if any congruence relation on $L$ is uniquely determined by its restriction to $S$; see [5]. The reader should have little difficulty in deriving the following statement.

Corollary 3. Let $L$ be a semimodular lattice and $C$ be a finite maximal chain in $L$. Then $C$ is a congruence-determining sublattice of $L$.

\section{REFERENCES}

[1] G. Birkhoff, Lattice Theory, third edition, Amer. Math. Soc., Providence, R.I., 1967.

[2] R. Dedekind, Über die von drei Moduln erzeugte Dualgruppe, Math. Annalen 53 (1900), 371-403. Reprinted in Gesammelte mathematische Werke, Vol. 2, pp. 236-271, Chelsea, New York, 1968.

[3] R. Dedekind, Gesammelte mathematische Werke. Bände I-III. (German) Herausgegeben von R. Fricke, E. Noether und O. Ore Chelsea Publishing Co., New York 1968 Vol. I: iii+397 pp.; Vol. II: iv+442 pp.; Vol. III: iii+223-508 pp.

[4] G. Grätzer, General Lattice Theory, second edition. New appendices by the author with B. A. Davey, R. Freese, B. Ganter, M. Greferath, P. Jipsen, H. A. Priestley, H. Rose, E. T. Schmidt, S. E. Schmidt, F. Wehrung, and R. Wille. Birkhäuser Verlag, Basel, 1998. xx+663 pp. ISBN: 0-12-295750-4; 3-7643-5239-6 Softcover edition, Birkhäuser Verlag, Basel-Boston-Berlin, 2003. ISBN: 3-7643-6996-5 Reprinted July, 2007.

G. Grätzer, 
A NEW LOOK AT THE JORDAN-HÖLDER THEOREM FOR SEMIMODULAR LATTICES 3

[5] The Congruences of a Finite Lattice, A Proof-by-Picture Approach. Birkhäuser Boston, 2006. xxiii+281 pp. ISBN: 0-8176-3224-7.

[6] N. Jacobson, Basic algebra. I. Second edition. W. H. Freeman and Company, New York, 1985. xviii+499 pp. ISBN: 0-7167-1480-9

[7] J.B. Nation, Notes on Lattice Theory, 1991, http://www.math.hawaii.edu/ jb/books.html

[8] H. Wielandt, Eine Verallgemeinerung der invarianten Untergruppen, Math. Zeit. 45 (1939), 209-244.

Department of Mathematics, University of Manitoba, Winnipeg, MB R3T 2N2, Canada E-mail address, G. Grätzer: gratzer@ms.umanitoba.ca

URL, G. Grätzer: http://server.math.umanitoba.ca/homepages/gratzer/

University of Hawaini, Honolulu, Hi, USA

E-mail address, J.B. Nation: jb@math.hawaii.edu 